\title{
Cultural Selection and Genetic Diversity in Humans
}

Hal Whitehead

Department of Biology, Dalhousie University, Halifax

Peter J. Richerson

Department of Environmental Science and Policy, University of California, Davis

Robert Boyd

Department of Anthropology, University of California, Los Angeles

Running title: Cultural Selection and Genetic Diversity in Humans

July 3, 2002 


\begin{abstract}
Recent research into human origins has largely focused on deducing past events and processes from current patterns of genetic variation. Some human genes possess unexpectedly low diversity, seemingly resulting from events of the late Pleistocene. Such anomalies have previously been ascribed to population bottlenecks or selection on genes. For four species of matrilineal whale, evidence suggests that cultural evolution may have reduced the diversity of genes which have similar transmission characteristics to selective cultural traits, through a process called cultural hitchhiking. Cultural evolution is characteristic of human societies and so should be considered as a potential determinant of human genetic diversity. A stochastic simulation of gene and cultural dynamics in an array of hunter-gatherer tribes shows that cultural selection has the potential to severely reduce genetic diversity if: inter-tribe gene flows are reasonably low (< 0.6-15 genes/tribe/generation); cultural evolution changes fitness by $>\sim 0.3-3 \%$ /generation; and fitness is changed more by cultural innovation within a tribe than cultural assimilation from neighboring tribes. Thus cultural hitchhiking may explain low diversity and short coalescence times in mitochondrial and, especially, Y-linked human genes.
\end{abstract}

Key words: Culture; Evolution; Human origins; Genetic diversity; Cultural group selection; Geneculture coevolution 


\section{INTRODUCTION}

Recent research into human origins has largely focused on deducing past events and processes from current patterns of genetic variation (Bertranpetit 2000; Harpending et al. 1998). While there is general agreement from these studies that anatomically modern humans evolved in Africa very roughly 200,000 years ago, a number of unexpected results have emerged (Disotell 1999). They include a quite recent common mitochondrial DNA (mtDNA), female, ancestor (Cann et al. 1987; Vigilant et al. 1991). Our common Y-chromosome, male, ancestor seems even more remarkably recent (Seielstad et al. 1999; Shen et al. 2000; Thomson et al. 2000; Underhill et al. 2000). Compared with other apes, we have, despite our large current population, relatively low effective mtDNA and Y-chromosome population sizes (Gagneux et al. 1999; Stone et al. 2002) and a low ratio of mtDNA diversity to nuclear heterozygosity (Wise et al. 1997). Furthermore, different patterns of human evolution are suggested by different genetic markers or by the same marker in different regions (Harpending and Rogers 2001; Harris and Hey 1999). For instance, there is a lack of evidence for population expansion in some modern hunter-gatherers, but an expansion, clearly evident in the patterns of genetic variation of other groups of humans, occurred when all, or almost all, humans were hunter-gatherers (Excoffier and Schneider 1999). Scientists have tried to explain such anomalous results in a number of ways, especially invoking population bottlenecks or selection on genes (Harding 1999; Harpending and Rogers 2001; Harpending et al. 1998). However, these explanations are not always satisfactory (Harding 1999; Harpending and Rogers 2001) and Harding (1999) suggests that a different perspective and new models of human origins may be needed.

A potentially important factor is gene-culture coevolution. It is clear that, in humans, genes and culture have coevolved (Feldman and Laland 1996), but this is at most briefly alluded to in most recent discussions of the genetic evidence for human origins. Cavalli-Sforza and his colleagues argue that cultural innovations have led to expansions of some human populations, which left clear imprints on the current geography of human genes (Cavalli-Sforza et al. 1993, 1994). Concentric contours of gene frequency surround the sites of major cultural innovations which gave certain groups of humans selective advantages, and so allowed them to expand at the expense of neighboring populations (Cavalli-Sforza et al. 1993, 1994). Archaeological and linguistic evidence suggest that, in pre-agricultural hunter-gather populations, cultural innovations triggered the spreads of populations of humans in which the innovations occurred (Bettinger and Baumhoff 1982). Such events may have affected other attributes of human population genetics in addition to geographic pattern.

In four species of whale with remarkably low mtDNA diversity, Whitehead (1998) has suggested that matrilineally-transmitted cultural innovations may have given certain matrilineal groups a selective advantage, allowing them to outcompete other groups, and so eventually reducing mtDNA variation in the population. When neutral or nearly-neutral genes and selectively advantageous cultural traits are being transmitted in parallel (Asymmetrically $\cong$ in the terminology of Boyd and Richerson 1985) genetic diversity may be reduced by this process which has been called Acultural hitchhiking (Whitehead 1998) as it is analogous to genetic hitchhiking (in which a selectively advantageous gene reduces the diversity of its linked neighbor; Maynard Smith and Haigh 1974).

Here, we explore whether cultural hitchhiking might have been responsible for some of the 
apparent anomalies in human genetic variation. These anomalies seem to date from times $(\sim 10,000-$ $200,000 \mathrm{ybp}$ ) when most, if not all, humans were hunter-gatherers. Therefore, the potential for cultural hitchhiking is examined within the context of what is known of late Pleistocene hunter-gatherer societies. In contrast to the matrilineal, sympatric whale societies modeled in the first exploration of cultural hitchhiking (Whitehead 1998), late Pleistocene hunter-gatherers seem to have been largely patrilineal and territorial, although there was likely considerable variation between places and with time (Lewin 1998).

Richerson and Boyd (1998) argue that strong cultural structuring of human populations has been essential in the evolution of human ultrasociality, and suggest that a substantial proportion of recent human evolution has been governed by competition for resources among culturally homogeneous human groups. Soltis et al. (1995) use both theoretical considerations and empirical data to conclude that cultural group selection should be considered as a potentially important evolutionary force in human populations. Cultural group selection within human populations also forms an underlying theoretical framework for some anthropologists and macro-sociologists (Lenski et al. 1995). Our model follows this approach, and includes tribe-based demography with extinctions, competition for resources between tribes, intertribe flows of genes and culture, genetic mutation and cultural evolution. The model is a special case of the general models of genetic variation in subdivided populations described by Whitlock and Barton (1997), with the important additions of inter-tribe competition for resources and the dynamics of fitness change caused by cultural innovation. By varying the demographic, genetic and cultural parameters of the model, it is possible to outline the conditions under which cultural hitchhiking might have reduced the genetic diversity of a population of late Pleistocene hunter-gatherers. In any population, genes with distinct transmission characteristics (mitochondrial, Y-linked, X-linked, or autosomal) may have different effective population sizes and inter-tribe movement rates. Thus, the model is general with respect to gene type, allowing the likelihood of cultural hitchhiking to be assessed for different genes depending on their effective population sizes and movement rates.

\section{THE MODEL}

The model used is a stochastic simulation of gene and cultural dynamics in an array of tribes, each of which is homogeneous culturally but not necessarily genetically.

\section{The habitat}

Tribes are arranged on an $s$ by $s$ square grid of habitats. Thus there are $s^{2}$ tribes, and each has a maximum of eight neighboring tribes (diagonal neighbors are included). Each square habitat, $i$ (to simplify notation, a single suffix is used to represent elements of a square array), has a fixed (through time) resource availability $u_{i}$ (the equilibrium population that can be supported by these resources). Given an initial mean population size (of genes) per tribe, $P$, the $\left\{u_{i}\right\}=$ s are randomly produced by a log-normal distribution with mean $P$ and standard deviation 1.5P. The potential resources available to the tribe on habitat $i$ are: 


$$
U_{i}=u_{i}+f \cdot \sum_{j \in M(i)} u_{j}
$$

where $M(i)$ are the neighboring habitats to habitat $i$, and $f$ describes the relative importance of resources in neighboring habitats compared with a tribe $=\mathrm{s}$ own habitat. With low $f$, tribes depend principally on the resources within their own habitat, and there is little competition. As $f$ rises, so does the competition between neighboring tribes.

At generation $t(t=1 \ldots T)$, there are $n(i, t, x)$ copies of genotype $x$ on habitat $i$, so that the population of genes of the tribe on habitat $i$ is $p(i, t)=\Sigma n(i, t, x)$. When considering an autosomal locus, then $p$ will be approximately twice the effective population size of the tribe; for mtDNA or Y-loci, $p$ will be the effective number of breeding females or males respectively. The following events take place at each simulated generation: inter-tribe migration, cultural evolution, cultural assimilation, reproduction, and genetic mutation.

\section{Inter-tribe migration}

At the start of each generation, a number of genes are exchanged between neighboring tribes. If $m$ $(0<m<1)$ is roughly the rate of gene flow into or out of a tribe per generation (the true rate, $m=$, was calculated from runs of the model), the number of genes of type $x$ moving from tribe $i$ to neighboring tribe $j$ is:

$$
g(i, j, x)=m / 8 \cdot n(i, t, x) \cdot \sqrt{\frac{p(j, t)}{p(i, t)}}
$$

This formulation means that the number of migrants between neighboring tribes is proportional to the geometric mean of their population sizes, that there is no net change in tribe population sizes [as $\Sigma$ $g(i, j, x)=\Sigma g(j, i, x)=m / 8 . /(p(i, t) . p(j, t))]$, and that proportionally more breeders move from small tribes, and fewer from large tribes, as in Birdsell=s (1966) suggestion for Pleistocene hunter-gatherers. In the very rare cases where application of equation 2 resulted in more genes moving from a tribe than were present in it, it is replaced by:

$$
g(i, j, x)=n(i, t, x) \cdot \frac{\sqrt{\frac{p(j, t)}{p(i, t)}}}{\sum_{k \in M(i)} \sqrt{\frac{p(k, t)}{p(i, t)}}}
$$


In these cases, migration is asymmetric, with more genes moving into the very small tribe than out of it.

\section{Cultural evolution}

Each tribe, and all its members, have a culturally-determined fitness $w(i, t)$, which specifies how well the tribe competes with neighboring tribes for the available resources $\left(U_{i}\right)$. Cultural evolution is defined by two parameters, the frequency (?) and magnitude (s) of cultural innovations (which are assumed to spread through a tribe within a generation), so that:

$$
w(i, t+1)=\begin{gathered}
w(i, t) \text { with probabilit } \mathrm{y} 1-\rho \\
|w(i, t) . \mathrm{N}(1, \sigma)| \text { with probabilit y } \rho
\end{gathered}
$$

where $\mathrm{N}(1, \mathrm{~s})$ is a normal random variable with mean 1.0 and standard deviation s. Thus, roughly once every $1 /$ ? generations, a tribe produces a cultural innovation which changes its fitness by a factor with mean 1.0 and standard deviation s. In this formulation, the approximate significance of the cultural innovations is given by s (high s indicating that cultural innovations have a major effect on fitness), and cultural innovations can have positive or negative effects on fitness. The relative incidence and importance of negative, as opposed to positive, innovations, is unknown. Therefore, the model was also run with a modification so that all innovations had a positive effect on fitness (in equation 4, the new fitness when there is an innovation is given by $w(i, t) .(1+|\mathrm{N}(0,1.67 . \mathrm{s})|)$ (which has standard deviation $w(i, t) . \mathrm{s})$, rather than $|w(i, t) . \mathrm{N}(1, \mathrm{~s})|)$.

\section{Cultural assimilation between neighboring tribes}

Cultural assimilation between neighboring tribes was added in some runs of the model. At each generation, each tribe, $i$, has a probability a of receiving cultural input from its most culturally advanced neighboring tribe:

$$
w(i, t+1)=\begin{gathered}
w(i, t) \text { with probabilit } \mathrm{y} 1-\alpha \\
(1-\beta) \cdot w(i, t)+\beta \cdot \max [w(j, t): j=i, j \in M(i)] \text { with probabilit } \mathrm{y} \alpha
\end{gathered}
$$

Thus, $a$ is the frequency of cultural assimilation, and $B$ its magnitude.

\section{Reproduction and extirpation}

Neighboring tribes compete for resources, so that the ability of tribe $i$ to exploit resources is proportional 
to the product of its population size and its fitness, $w(i, t) \cdot p(i, t)$. Then the total pressure on $U_{i}$, the resources being exploited by tribe $i$, is $w(i, t) \cdot p(i, t)+f . S w(j, t) . p(j, t)$ [summing over neighboring tribes], and a particular gene receives an average of $w(i, t) \cdot U_{i} /[w(i, t) \cdot p(i, t)+f \cdot S w(j, t) \cdot p(j, t)]$ resources which determine its contribution to the next generation. So the number of genes of a given type $x$ in tribe $i$ in the following generation is given by:

$$
n(i, t+1, x)=\operatorname{Po}\left[\frac{n(i, t, x) \cdot w(i, t) \cdot U_{i}}{w(i, t) \cdot p(i, t)+f \cdot \sum_{j \in M(i)} w(j, t) \cdot p(j, t)}\right]
$$

where $\operatorname{Po}(z)$ is a Poisson variable with mean $z$. In this model, if all tribes have equal fitness, then their populations, $\{p(i, t)\}$, approximate the resource values of their habitats, $\left\{u_{i}\right\}$. However, a tribe surrounded by fitter neighbors will tend to shrink, while one with a cultural fitness advantage will tend to grow, processes for which there is some empirical justification in the anthropological literature (Soltis et al. 1995).

If, after inter-tribe migration (equation 2) and reproduction (equation 6), a tribe is extirpated $(p(i, t)=0)$, then its habitat is repopulated by the neighboring tribe with the highest net fitness $[p(j, t)$. $w(j, t)]$, with $1 / 9$ of the population of the source tribe moving to the empty habitat, and proportional numbers from each genotype in the source tribe. The initial cultural fitness of the new tribe is that of the source tribe, but, in subsequent generations, their cultures evolve independently. This part of the model is a substantial simplification of the varied scenarios in which tribes could supplant each other from a habitat. However, it contains elements which are both essential to the process of cultural hitchhiking and consistent with the ethnographic evidence for primitive societies: Adefeated $\cong$ tribes were often either extinguished, forced to migrate from their habitat, or, if members were assimilated into surrounding tribes, they tended to have low reproductive success; and new tribes were formed by fission of successful tribes (Soltis et al. 1995).

\section{Mutation}

Each gene in each generation has probability $\mu$ of mutating into a totally new genotype (different at one base pair from the old genotype, when considering mtDNA). There are no back mutations.

\section{Procedure for running model}

For any set of non-cultural model parameters $(s, f, m, \mu, P)$, the resource availabilities of the habitat, $\left\{u_{i}\right\}$, were simulated as described above $(\log$-normal $(P, 1.5 P))$. Then the initial gene population of each tribe was randomly chosen to approximately equal the resource availability of its habitat, as given by $p(i, 1)=\mathrm{N}\left(u_{i}, 0.1 . u_{i}\right)$. Initially all individuals were genetically identical, and all tribes had a cultural fitness 
$(w(i, 1))$ of 1 . The model was then run without cultural evolution (?=0) until, at $T_{C}$ generations, the overall genetic diversity of the entire population first exceeded the expected equilibrium genetic diversity given an infinite allele model (using formulae from Birky et al. 1983), and then for another $T_{C}$ generations to produce a population with a genetic structure close to that expected at drift-mutation equilibrium. At this stage, the genetic structure of the culturally undifferentiated population (given by $\left\{n\left(i, 2 T_{C}, x\right)\right\}$ ) was saved.

To investigate cultural hitchhiking, each of these populations was then run 100 times for 200 further generations, under a variety of modes of cultural evolution (determined by a, $\beta, ?$ and s).

The significance of cultural hitchhiking for a particular set of population parameters was evaluated by the change in genetic diversity of the whole population over the 200 test generations averaged over the 100 runs. The proportion of model runs with a particular set of parameters in which genetic diversity was reduced by $50 \%$ or more was used to indicate the probability of cultural hitchhiking. Nucleotide diversity was also examined for the case of mtDNA, but results were similar to those for haplotype/genotype diversity and are not presented here.

The model was constructed and run using MATLAB5.2.

\section{Parametrization}

Initial non-cultural parameters were $s=6$ (i.e. 36 tribes); $f=0.2 ; P=20,60$, or 180 genes/tribe; $m=0.01$, 0.04 , or 0.16 genes/tribe/generation; $\mu=2.10^{-4} /$ generation (or $10^{-6}$ at 200 base pairs for mitochondrial DNA, except $\mu=2.10^{-3} /$ generation for $P=20$ to give reasonable population genetic diversity). These represent a total initial population varying from about $720-6,500$ gene copies $\left(\sim s^{2} . P\right)$. In each of these scenarios, 10 modes of cultural evolution were examined with no cultural assimilation between tribes $(\mathrm{a}=0): ?=0$ (control, no cultural evolution), and all combinations of $?=0.025,0.05,0.10$ and $\mathrm{s}=0.02,0.1$, 0.5 . The same set of parameter combinations were used in an environment where all cultural innovations were positive. Cultural assimilation was then added both to the initial model and that with just positive innovations $\left(\mathrm{a}=0.01,0.02,0.04,0.08\right.$ and $0.16 ; \beta=0.2,0.5$ and $1.0 ; s=6 ; f=0.2 ; \mu=2.10^{-4}$ /generation; $P=60 ; m=0.01 ; ?=0.025$ (just positive innovations) or 0.10 (positive/negative innovations) and $\mathrm{s}=0.5$ ). Additional runs of the model tested its sensitivity to parameter variation (see Appendix).

Actual rates of migration between neighboring habitats were found to be $m==0.72 m$ for the $6 x 6$ array, and $m==0.81 \mathrm{~m}$ for a $10 \times 10$ array (the difference being as expected given the larger proportion of edge habitats with fewer neighbors in the smaller array).

\section{RESULTS}

Runs of the model showed that reduction of genetic diversity was a frequent, but not always predictable, consequence of cultural evolution under some parameter combinations. The reduced diversity is consistent with Whitlock and Barton=s (1997) general result that variation in reproductive success among demes in a structured population decreases genetic diversity. The reduction in diversity was little affected by changes in mutation rates or the number of tribes in the population, but was generally greater with increased competition among tribes for resources. Four factors accounted for most variation in the 
degree of reduction in genetic diversity (see Appendix): the cultural evolution rate (?.s, the product of the frequency and magnitude of cultural innovations); the mean number of genes transferring from a tribe in each generation $\left(N_{m}=P . m=\right.$, the product of the mean tribe size and the migration rate); whether cultural innovations could have negative effects on fitness; and the importance of cultural assimilation (indicated by the ratio of the sum of absolute changes in fitness caused by assimilation to those caused by innovation; this ratio was close to $0.47 . \mathrm{a} /$ ? s).

With all sets of parameters and variants investigated (except cultural assimilation), there was a substantial (>50\%) average decrease in genetic diversity after 200 generations of cultural evolution if the cultural evolution rate was greater than about 0.013 /generation, and less than a mean of 0.5 gene transferred out of a tribe per generation (Fig. 1). With just positive innovations, these conditions were relaxed to cultural evolution rates greater than about 0.005 /generation and gene transfer rates less than about 3/generation. Adding cultural assimilation among neighboring tribes to the model had little effect when less than $25 \%$ of fitness change was caused by assimilation (Fig. 2). However, when the assimilation rate was greater than the innovation rate, there was much less of a reduction in genetic diversity (Fig. 2). For genetic diversity to be substantially (>50\%) reduced within 200 generations with high probability, then inter-tribe gene flows had to be lower than $\sim 0.6$ genes/tribe/generation, cultural evolution had to change a tribe $=\mathrm{s}$ fitness by more than $\sim 2.8 \%$ /generation, and cultural assimilation needed to be considerably less important than cultural innovation (Table 1). However, cultural hitchhiking could still occur, but less reliably, when these conditions were relaxed to mean inter-tribe gene flows of up to $\sim 15$ genes/tribe/generation, cultural evolution rates as low as $0.3 \% / g e n e r a t i o n$, and with assimilation being up to twice as important as innovation (Table 1). If all innovations had a positive effect on fitness, then genetic diversity was more easily reduced (Fig. 1, Table 1).

In these simulations, the results of cultural hitchhiking were unpredictable, even with an initial population structure and set of parameters. For instance, using a set of parameters which gave substantial cultural hitchhiking ( $P=60 ; \mathrm{s}=6 ; f=0.2 ; \mu=10^{-4} /$ generation; $m=0.01 ; ?=0.05$ and $\mathrm{s}=0.5$ ), the mean reduction in genetic diversity was $79 \%$. However, for 21 of the 100 runs of the model with identical initial population structures the reduction in diversity after 200 generations was less than 50\%, and for 62 runs it was greater than $90 \%$.

\section{DISCUSSION}

In one respect the model is conservative, as runs only considered 200 generations, or perhaps 4,000 years, and conditions suitable for cultural hitchhiking could have persisted over much greater time periods. Human cultural evolution generally decelerates as we look back through prehistory and history (Klein 1999), so that there is probably some point in the past before which the rate of cultural evolution was too low for cultural hitchhiking to be feasible (Fig. 1). Conversely, as cultures evolved, there were increases in both tribe sizes and migration rates because of developments such as horticulture and the use of animals for transport (Lenski et al. 1995), so increasing the inter-tribe gene flow, and reducing the likelihood of cultural hitchhiking (Fig. 1). Thus, there may have been a window in human prehistory (perhaps different in different geographical areas) for cultural hitchhiking, when cultures were evolving sufficiently fast but had not yet led to a substantial increase in inter-tribe gene flows. We do not know 
how long this window was (or even if it really existed), so it is perhaps appropriate that we were conservative in just considering 200 generations.

The model is based upon what we can reasonably infer about human societies in the late Pleistocene. It assumes cultural evolution, that cultural traits affect fitness, and that culturally advanced tribes outcompete and replace others, processes for which there is abundant evidence. Like all other models of natural systems, this model is a simplification. However, substantial modifications to the model and its parameters (see Appendix) caused only quite small changes to the general conditions under which cultural hitchhiking occurred, indicating that somewhat similar conditions may have regulated the effects of cultural evolution on genetic diversity among prehistoric humans.

So, are these conditions likely to have been operating among human populations of the late Pleistocene? Despite considerable uncertainty, and undoubtedly great variability (Kelly 1995), it is generally assumed, following Birdsell (1966), that mean tribe sizes were about 500 individuals, with an effective population size of approximately 175. This translates into mean tribal gene populations of about 87 for mtDNA and Y-linked genes, 257 for X-linked genes and 350 for autosomal genes. Tribes of hunter-gatherers are, and were, very largely patrilineal with little transfer of breeding males between tribes. In contrast, breeding females do transfer. Tindale (1953) estimated the mean rate of inter-tribe marriages among Australian aboriginals to be about $15 \%$, principally females transferring to neighboring tribes. These data suggest rates of inter-tribe gene flow per generation of about $13 \mathrm{mtDNA}$ genes, $<1$ Y-linked gene, $26 \mathrm{X}$-linked genes, and 26 autosomal genes. This implies that the first condition for cultural hitchhiking, low gene transfer rates, is likely satisfied for Y-linked genes, but a reduction in diversity is less likely for mtDNA, and unlikely for autosomal and $\mathrm{X}$-linked genes. However, Tindale $=\mathrm{s}$ inter-tribal marriage rate of $15 \%$ is high compared with results on other modern hunter-gatherers (e.g. Hill and Hurtado 1996), and if it is also higher than occurred in the late Pleistocene, then cultural hitchhiking with autosomal or X-linked genes may have been feasible. These arguments should be considered within the context of considerable debate about how similar the societies of current or recent human-gatherers are to those of the late Pleistocene (Lewin 1998; O'Connell 1999).

The second condition for cultural hitchhiking is that cultural evolution must have been reasonably rapid, changing mean fitness by more than about $0.3-2.8 \%$ per generation. This is undoubtedly true for recent humans, but as noted above, it is unclear how far back in pre-history the condition holds.

The final principal restriction on cultural hitchhiking is that inter-tribe cultural assimilation rates must be reasonably low. There is some evidence that it is low in current aboriginal populations. Among African societies, demic diffusion — the movement of people with distinctive cultures — seems to have been a more important determinant of most cultural practices than cultural assimilation (Guglielmino et al. 1995; Hewlett et al. 2002). The metapopulation model used in this paper employs a form of cultural diffusion.

What kinds of cultural innovations could produce the effects described by the model? Much attention has been devoted to those that involved objects and left archaeological traces, such as fire, tools and weapons. These may have given their innovators substantial, and sometimes overwhelming, fitness advantages, but they also occurred rarely and may have had high assimilation rates between tribes.

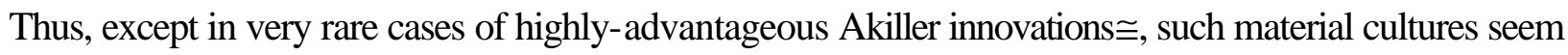
unlikely to have driven cultural hitchhiking. Better candidates may be those concerning social structure 
and foraging strategies. These commonly vary between tribes, show substantial evolution, can result in substantial differences in fitness, especially when different types are in competition (Bettinger and Baumhoff 1982), but, because they generally relate to communal behavior, are less easily assimilated and tend to be stable over many generations (Hewlett et al. 2002).

In conclusion, given what is known about late Pleistocene human societies, it is possible that cultural hitchhiking has substantially reduced the diversity of some genes. The Y-chromosome markers are especially good candidates because of the low rates of transfer between cultural tribes, and they show particularly low diversity and short coalescence times (Seielstad et al. 1999; Shen et al. 2000; Thomson et al. 2000; Underhill et al. 2000). However, cultural hitchhiking may have also contributed to the low diversity of human mtDNA (Gagneux et al. 1999; Vigilant et al. 1991). We can envisage the cultural hitchhiking occurring over virtually the entire human population at a time when it was reasonably

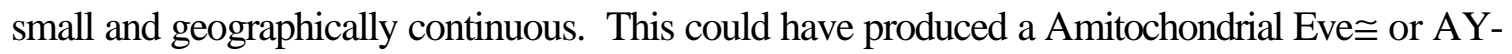
chromosome Adam€ who possessed, and whose descendants possessed and perhaps accumulated, cultural attributes conferring fitness advantages. Cultural hitchhiking could also have been important for segments of the human population. For instance, episodes of relatively recent cultural hitchhiking, rather than population bottlenecks, might have reduced mtDNA diversity in some populations of current huntergatherers, so masking the signals of earlier population expansion which Excoffier and Schneider (1999) expected but did not find. The model shows that the effects of cultural hitchhiking are unpredictable, perhaps explaining some of the discordances between loci and geographical regions in recent studies on human genes (Harpending and Rogers 2001).

A variety of statistics and displays, including frequency spectra of mutations and mismatch distributions, have recently been used to infer human evolutionary history from genetic data (Harpending et al. 1998). It would be useful to examine how they are affected by cultural hitchhiking. Perhaps even more valuable for assessing the feasibility of cultural hitchhiking in human evolution would be improved knowledge of inter-tribe gene and cultural flows among late Pleistocene hunter-gatherers, as well as the processes and results of inter-tribe competition.

Our model does not prove that cultural hitchhiking has reduced human genetic diversity. However, it suggests that cultural evolution and cultural selection should be considered along with population bottlenecks and genetic selection as a potential cause of anomalously low genetic diversity. Cultural evolution and cultural selection are known and important elements in the emergence of modern humans, so that, in contrast to explanations for low diversity invoking population bottlenecks or selection on what were previously thought to be neutral genes, no new factor is invoked.

\section{ACKNOWLEDGMENTS}

Thanks to Leah Gerber for library research, Kim Hill for information on hunter-gatherer demography, and Henry Harpending, Robert Latta, Luke Rendell and two anonymous reviewers for important suggestions for improving the manuscript. The study was funded by the Natural Sciences and Engineering Research Council of Canada. 


\section{REFERENCES}

Bertranpetit, J. (2000). Genome, diversity, and origins: The Y chromosome as a storyteller. Proceedings of the National Academy of Sciences of the United States of America 97, 6927-6929.

Bettinger, R.L., \& Baumhoff, M.A. (1982). The Numic spread: Great Basin cultures in competition. American Antiquity 47, 485-503.

Birdsell, J.B. (1966). Some predictions for the Pleistocene based on equilibrium systems among recent hunter-gatherers. In R.B. Lee \& I. DeVore (Eds.), Man the Hunter, (pp.229-240). Chicago: Aldine.

Birky, C.W., Maruyama, T., \& Fuerst, P. (1983). An approach to population and evolutionary genetic theory for genes in mitochondria and chloroplasts, and some results. Genetics 103, 513-527.

Boyd, R., \& Richerson, P. (1985). Culture and the evolutionary process. Chicago: Chicago University Press.

Cann, R.L., Stoneking, M., \& Wilson, A.C. (1987). Mitochondrial DNA and human evolution. Nature 325, 31-36.

Cavalli-Sforza, L.L., Menozzi, P., \& Piazza, A. (1993). Demic expansions and human evolution. Science 259, 639-646.

Cavalli-Sforza, L.L., Menozzi, P., \& Piazza, A. (1994). The history and geography of human genes. Princeton, NJ: Princeton University Press.

Disotell, T.R. (1999). Human evolution: origins of modern humans still look recent. Current Biology 9, R647-650.

Excoffier, L., \& Schneider, S. (1999). Why hunter-gatherer populations do not show signs of Pleistocene demographic expansions. Proceedings of the National Academy of Sciences of the United States of America 96, 10597-10602.

Feldman, M.W., \& Laland, K.N. (1996). Gene-culture coevolutionary theory. Trends in Ecology and Evolution 11, 453-457.

Gagneux, P., Wills, C., Gerloff, U., Tautz, D., Morin, P.A., Boesch, C., Fruth, B., Hohmann, G., Ryder, O.A., \& Woodruff, D.S. (1999). Mitochondrial sequences show diverse evolutionary histories of African hominids. Proceedings of the National Academy of Sciences of the United States of America 96, 5077-5082.

Guglielmino, Viganotti, C.R.C., Hewlett, B., \& Cavalli-Sforza, L.L. (1995). Cultural variation in Africa: role of mechanisms of transmission and adaptation. . Proceedings of the National Academy of Sciences of the United States of America 92, 7585-7589.

Harding, R.M. (1999). More on the X files. Proceedings of the National Academy of Sciences of the United States of America 96, 2582-2584.

Harpending, H., \& Rogers, A. (2001). Genetic perspectives on human origins and differentiation. Annual Review of Genomics and Human Genetics 1, 361-385.

Harpending, H.C., Batzer, M.A., Gurven, M., Jorde, L.B., Rogers, A.R., \& Sherry, S.T. (1998). Genetic traces of ancient demography. Proceedings of the National Academy of Sciences of the United States of America 95, 1961-1967. 
Harris, E.E., \& Hey, J. (1999). X chromosome evidence for ancient human histories. Proceedings of the National Academy of Sciences of the United States of America 96, 3320-3324.

Hewlett, B.S., de Silvestri, A., \& Guglielmino, C.R. (2002). Semes and genes in Africa. Current Anthropology 43, 313-321.

Hill, K., \& Hurtado, M.A. (1996). Ache life history: the ecology and demography of a foraging people. New York: Aldine de Gruyter.

Kelly, R.L. (1995). The foraging spectrum. Diversity in hunter-gatherer lifeways. Washington, DC: Smithsonian Institution Press.

Klein, R. (1999). The human career, 2nd ed. Chicago: University of Chicago Press.

Lenski, G., Nolan, P., \& Lenski, J. (1995). Human societies, 7th ed. New York: McGraw-Hill.

Lewin, R. (1998). New views emerge on hunters and gatherers. Science 240, 1146-1148.

Maynard Smith, J., \& Haigh, J. (1974). The hitch-hiking effect of a favourable gene. Genetics Research 23, 23-35.

O'Connell, J.F. (1999). Genetics, archaeology, and Holocene hunter-gatherers. Proceedings of the National Academy of Sciences of the United States of America 96, 10562-10563.

Richerson, P.J., \& Boyd, R. (1998). The evolution of human ultrasociality. In I. Eibl-Eibesfeldt \& F.K. Salter (Eds.), Indoctrinability, ideology and warfare, (pp.71-95). London: Berghahn Books.

Seielstad, M., Bekele, E., Ibrahim, M., Touré, A., \& Traoré, M. (1999). A view of modern human origins from Y chromosome microsatellite variation. Genome Research 9, 558-567.

Shen, P., Wang, F., Underhill, P.A., Franco, C., Yang, W.-H., Roxas, A., Sung, R., Lin, A.A., Hyman, R.W., Vollrath, D., Davis, R.W., Cavalli-Sforza, L.L., \& Oefner, P.J. (2000). Population genetic implications from sequence variation in four Y chromosome genes. Proceedings of the National Academy of Sciences of the United States of America 97, 7354-7359.

Soltis, J., Boyd, R., \& Richerson, P.J. (1995). Can group-functional behaviors evolve by cultural group selection? An empirical test. Current Anthropology 36, 473-494.

Stone, A.C., Griffiths, R.C., Zegura, S.L., \& Hammer, M.F. (2002). High levels of Y-chromosome nucleotide diversity in the genus Pan. Proceedings of the National Academy of Sciences of the United States of America 99, 43-48.

Thomson, R., Pritchard, J.K., Shen, P., Oefner, P.J., \& Feldman, M.W. (2000). Recent common ancestry of human Y chromosomes: evidence from DNA sequence data. Proceedings of the National Academy of Sciences of the United States of America 97, 7360-7365.

Tindale, N.B. (1953). Tribal and intertribal marriage among the Australian aborigines. Human Biology 25, 169-190.

Underhill, P.A., Shen, P., Lin, A.A., Jin, L., Passarino, G., Yang, W.H., Kauffman, E., Bonné-Tamir, B., Bertranpetit, J., Francalacci, P., Ibrahim, M., Jenkins, T., Kidd, J.R., Qasim Mehdi, S., Seielstad, M.T., Wells, R.S., Piazza, A., Davis, R.W., Feldman, M.W., Cavalli-Sforza, L.L., \& Oefner, P.J. (2000). Y chromosome sequence variation and the history of human populations. Nature Genetics 26, 358-361.

Vigilant, L., Stoneking, M., Harpending, H., Hawkes, K., \& Wilson, A.C. (1991). African populations and the evolution of human mitochondrial DNA. Science 253, 1503-1507.

Whitehead, H. (1998). Cultural selection and genetic diversity in matrilineal whales. Science 282, 1708- 
1711.

Whitlock, M.C., \& Barton, N.H. (1997). The effective size of a subdivided population. Genetics 146, 427-441.

Wise, C.A., Sraml, M., Rubinsztein, D.C., \& Easteal, S. (1997). Comparative nuclear and mitochondrial genome diversity in humans and chimpanzees. Molecular Biology and Evolution 14, 707-716. 


\section{APPENDIX}

In addition to the original set of parameters, and model variants, described in the Methods section, other parameter sets and variants of the model (all without cultural assimilation, $a=0$ ) were used to explore the sensitivity of the model, and the robustness of its results:

a) a variety of mutation rates (including $\mu=2.10^{-5}, 2.10^{-3}$ /generation; $s=6 ; \mathrm{P}=60 ; f=0.2 ; m=0.01$; $?=0.025,0.05,0.10$ and $\mathrm{s}=0.5)$;

b) larger tribes $\left(P=540 ; \mathrm{s}=6 ; f=0.2 ; \mu=2.10^{-4} /\right.$ generation; $m=0.01$; and $?=0.025,0.05,0.10$ and $\mathrm{s}=0.1$ and 0.5$)$;

c) a larger array of 100 square habitats $\left(s=10, f=0.2 ; \mu=2.10^{-4}\right.$ /generation; $P=60 ; m=0.01$; $?=0.025,0.05,0.10$ and $\mathrm{s}=0.5)$;

d) lower $(f=0.1)$ and higher $(f=0.3)$ levels of competition for resources between neighboring tribes $\left(s=6,10 ; \mu=2.10^{-4}\right.$ /generation; $P=60 ; m=0.01 ; ?=0.025,0.05,0.10$ and $s=0.5$ );

e) a lower level of connectivity between habitats, so that diagonally adjacent habitats are not considered neighbors, each tribe having a maximum of four neighboring tribes rather than 8 (and $\mathrm{A} 8 \cong$ is replaced by $\mathrm{A} 4 \cong$ in equation $2 ; s=6 ; f=0.2 ; \mu=2.10^{-4} /$ generation; $P=60 ; m=0.01$; $?=0.025,0.05,0.10$ and $\mathrm{s}=0.5$ ).

Some results of the original runs of the model and these additional ones are summarized in Figs 3-5. Fig. 3 indicates that, with a given set of non-cultural parameters and no cultural assimilation among tribes, the reduction in genetic diversity due to cultural hitchhiking is well expressed by the product of the frequency and magnitude of cultural innovations (?.s). Similarly, in Fig. 4, when cultural parameters are held constant, the effect of demographic parameters is quite well described by the mean number of genes transferring from a tribe in each generation $\left(N_{m}=P . m=\right.$, the product of the mean tribe size and the migration rate).

Most of the other changes to the model, or its parameters, made only moderate changes to the pattern of reduction in genetic diversity with cultural evolution rate (Fig. 5). Reductions in genetic diversity were similar whether there were 36 or 100 habitats, and whatever the mutation rate used. However, the reduction in genetic diversity was somewhat greater when inter-tribal competition for resources $(f)$ was increased, and somewhat less when tribes had fewer neighbors or inter-tribal competition was reduced (Fig. 5). 
Table 1. Ranges of model parameters required to obtain different rates of cultural hitchhiking (>50\% reduction in genetic diversity over 200 generations). In each case beneficial levels of the other parameters, as in column 3, are assumed.

\begin{tabular}{lcccc}
\hline & Innovations : & \multicolumn{3}{c}{ Cultural hitchhiking: } \\
Parameter: & $\begin{array}{c}>0 \% \text { of } \\
\text { runs }\end{array}$ & $\begin{array}{c}>50 \% \text { of } \\
\text { runs }\end{array}$ & $\begin{array}{c}>20 \% \text { of } \\
\text { runs }\end{array}$ \\
\hline $\begin{array}{l}\text { Gene transfers per tribe per } \\
\text { generation }(m=)\end{array}$ & Positive/negative & $<\sim 0.6$ & $<\sim 2$ & $<\sim 10$ \\
& Positive & $<\sim 1.0$ & $<\sim 5$ & $<\sim 15$ \\
$\begin{array}{l}\text { Cultural evolution rate per } \\
\text { generation (?.s) }\end{array}$ & Positive/negative & $>\sim 0.028$ & $>\sim 0.013$ & $>\sim 0.008$ \\
$\begin{array}{l}\text { Cultural assimilation rate } / \\
\text { Cultural innovation rate }\end{array}$ & Positive & $>\sim 0.013$ & $>\sim 0.008$ & $>\sim 0.003$ \\
& Positive/negative & $<\sim 0.25$ & $<\sim 0.5$ & $<\sim 2.0$ \\
& Positive & $<\sim 0.3$ & $<\sim 1.0$ & $<\sim 2.0$ \\
\hline
\end{tabular}




\section{Captions for Figures}

Figure 1. Contour surfaces of genetic diversity after 200 generations of cultural evolution in an array of hunter-gatherer tribes (as a proportion of its initial value) with the number of genes transferring between tribes per generation $\left(N_{m}\right)$ and the cultural evolution rate, for the case in which innovations could have positive or negative effects on fitness (left) and when effects were just positive (right) (parameter combinations as in Methods section, with no cultural assimilation between tribes).

Figure 2. Mean genetic diversity after 200 generations of cultural evolution (as a proportion of its initial value) plotted against the importance of cultural assimilation relative to cultural innovation in determining fitness, for the case in which innovations could have positive or negative effects on fitness $(>\forall=$, ?.s $=0.05 /$ generation) and when effects were just positive $(>+=, s=0.0125 /$ generation). (All simulations with $P=60 ; m=0.01 ; f=0.2 ; \mu=2.10^{-4} /$ generation.)

Figure 3. Mean genetic diversity after 200 generations of cultural evolution (as a proportion of its initial value) plotted against the rate of cultural evolution (?.s) for four frequencies of cultural innovation (?) (simulations with $s=6 ; P=60 ; f=0.2 ; m=0.01 ; \mu=2.10^{-4}$ /generation; all combinations of $?=0.025,0.05$, 0.10 and $\mathrm{s}=0.02,0.1,0.5,1.0)$.

Figure 4. Mean genetic diversity after 200 generations of cultural evolution (as a proportion of its initial value) plotted against the number of genes transferring out of, or into, a tribe per generation $\left(N_{m}\right)$ for different tribe sizes (simulations with $s=6 ; f=0.2 ; ? . s=0.05 /$ generation).

Figure 5. Mean genetic diversity after 200 generations of cultural evolution (as a proportion of its initial value) plotted against the rate of cultural evolution (?.s/generation) for the initial model (as specified in the methods section) and parameters $(>\forall=)$ or variants of it with: a lower (4 rather than 8 ) number of neighboring tribes $(>\mathrm{x}=)$; just positive cultural innovations $(>+=)$; lower $(>\leftrightarrow=, f=0.1)$ or higher $(>\uparrow=$, $f=0.3$ ) rates of intertribal competition for resources; and higher $\left(>\leftarrow=, \mu=2.10^{-3} /\right.$ generation $)$ and lower

( $>\uparrow=, \mu=2.10^{-5}$ /generation) mutation rates. Runs with 100 habitats $(s=10)$, rather than the standard 36 $(s=6)$, are shown by enlarged symbols. Points are slightly jittered so they do not overlay one another. (All simulations with $P=60 ; m=0.01$.) 
Positive/negative innovations

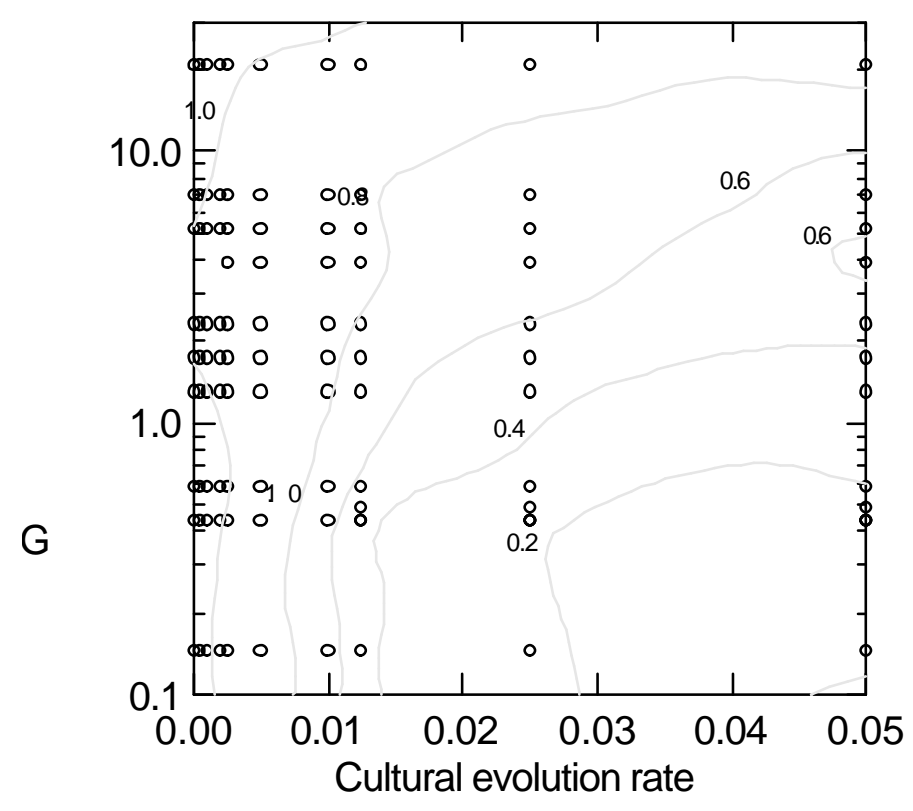

Positive innovations

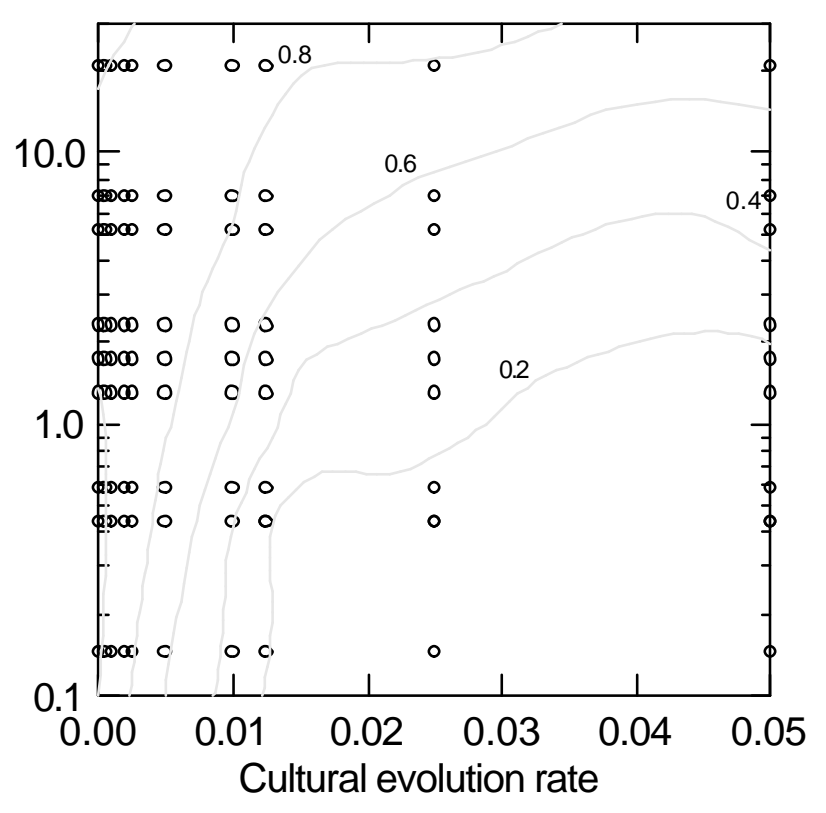

Figure 1 


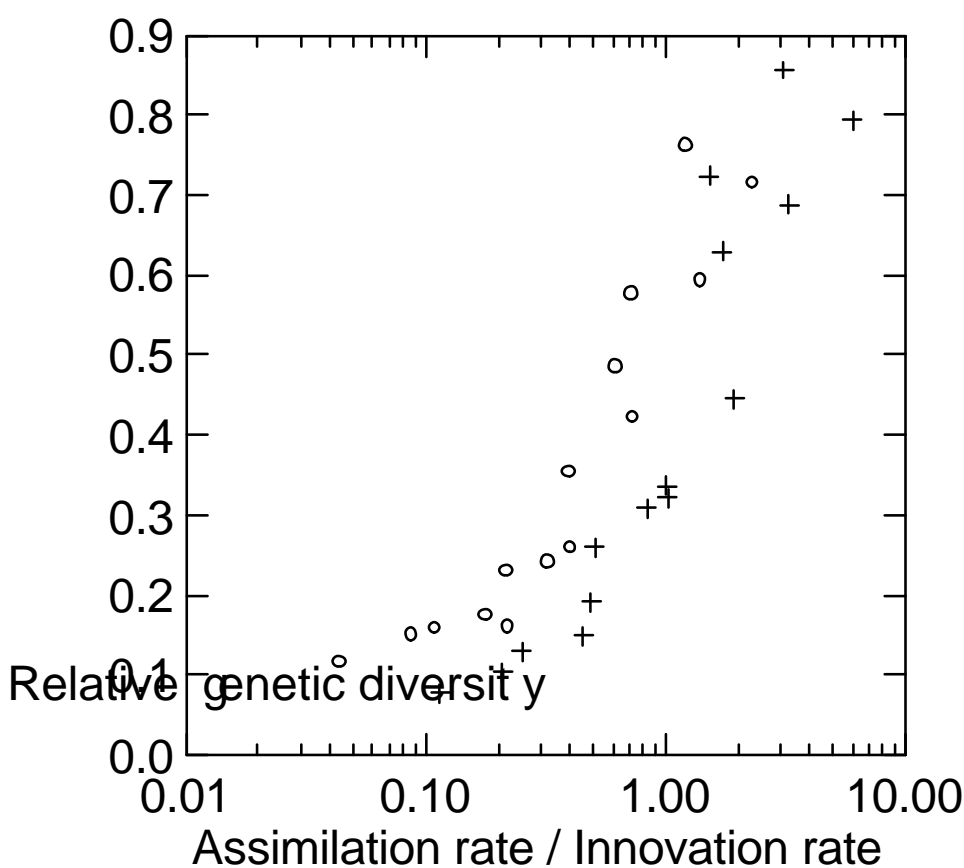

Innovations:

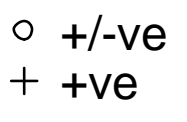

Assimilation rate / Innovation rate

Figure 2 


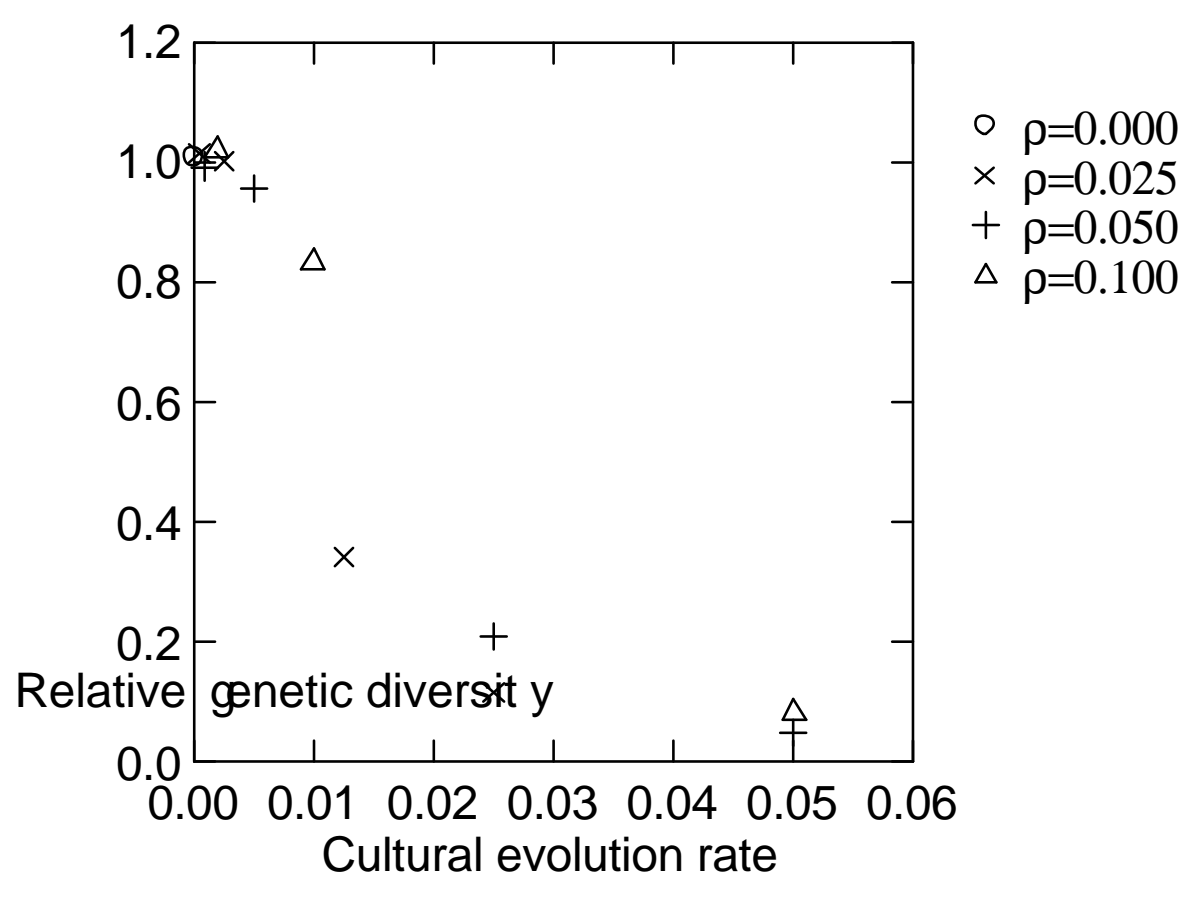

Figure 3 


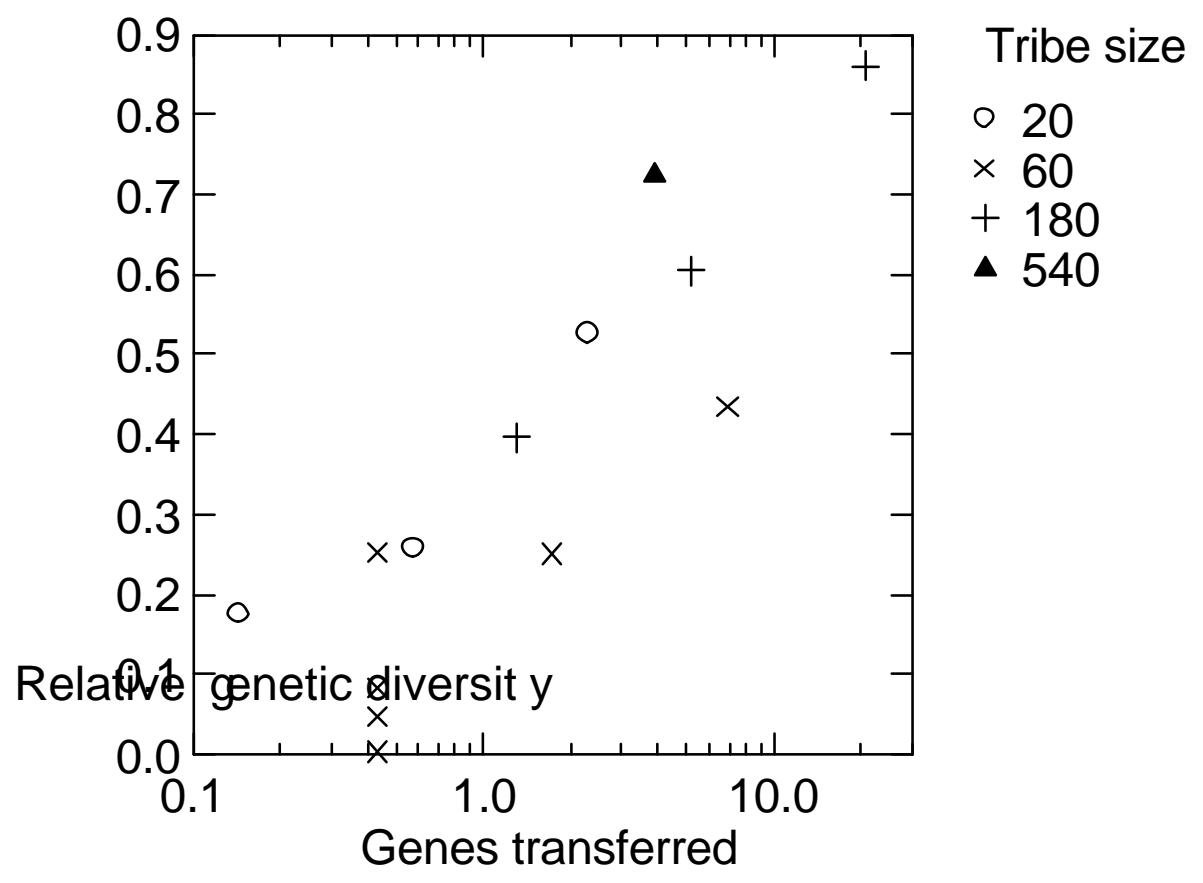

Figure 4 


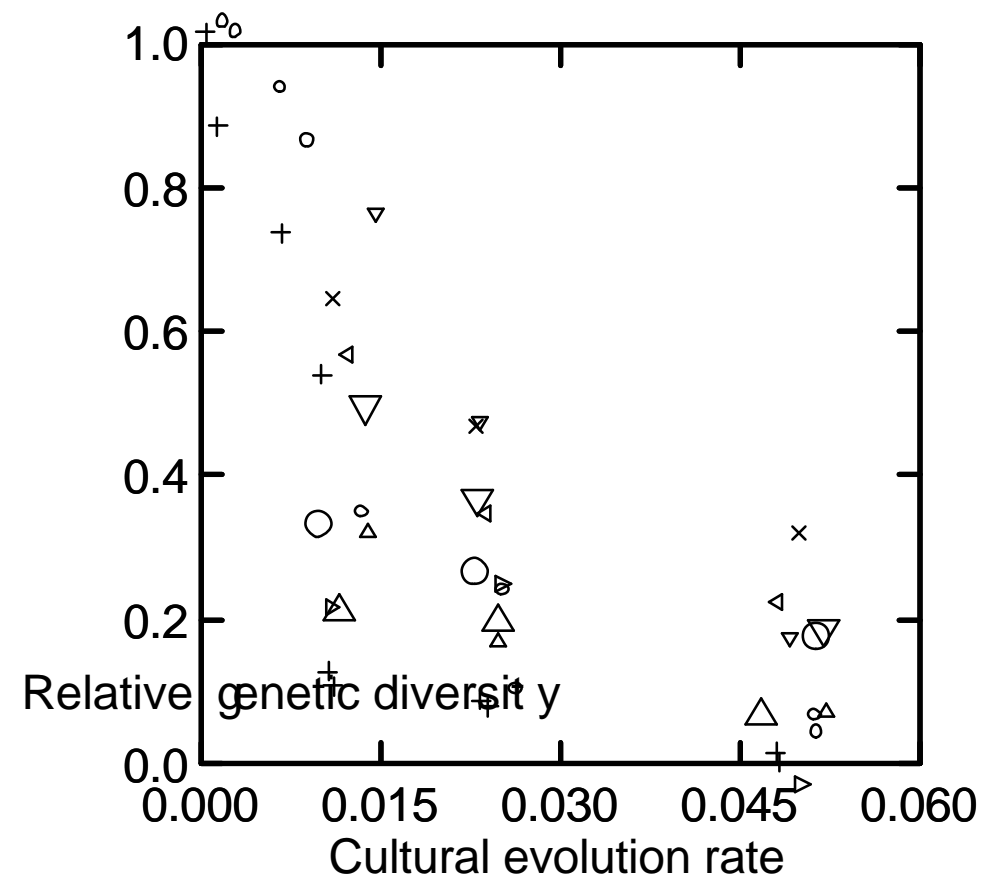

- Standard

$\times 4$ neighbours

+ +ve innovations

$\triangle f=0.3$

$\nabla f=0.1$

$\triangleleft \mu=0.002$

$\triangleright \mu=0.00002$

Figure 5 